\title{
ORIGIN OF METEORITES
}

$\mathrm{I}^{\mathrm{N}}$ the Halley Lecture* of 1940, Prof. F. A. Paneth presents a most stimulating and informative survey of the many hypotheses that have been proposed in the attempt to solve the difficult but fascinating problem of the origin of meteorites, with special reference to the evidence on which modern views are based.

In addition to the accepted groups of 'irons' and 'stones', Paneth follows Suess in claiming 'glasses' (tektites, etc.) as meteorites-despite the fact that no actual fall of tektites has been reliably observedon the ground that, since hypotheses explaining the occurrence of tektites as products of human manufacture or as terrestrial formations can easily be disproved, there is no alternative but to assume that they are of celestial origin. Moreover, the hypothesis of Michel, adopted by Lacroix, that tektites are formed in the atmosphere by the oxidation of silicon and other elements, is disproved by the fact that the rapid flight through the atmosphere of these alleged elements would allow far too little time for reaction with the enormous volume of oxygen that would be required. Paneth throws out the suggestion that silica-rich granitic bodies representing the original material of the tektites may have entered the Roche limit of the sun and there suffered melting and dispersal, to form swarms of glass globules on cooling after leaving the perihelion neighbourhood. It has, of course, to be recognized that our interpretations of the origin of iron and stone meteorites may not apply to the glasses. In this connexion it is relevant that while there are transitional varieties between the first two classes, there is none whatever between either of them and the third. In what follows, therefore, reference is made only to iron and stone meteorites.

Evidence consistent with the origin of meteorites in the solar system is: $(a)$ The close geochemical analogy between meteorites and terrestrial materials, even to the minor chemical elements and their distributions. (b) The identity in isotopic constitution of all the meteoritic elements so far tested with those of the corresponding terrestrial elements; this applies even to the radioactive elements uranium and potassium, and since the isotopic ratios of a radio-element vary with time, such identity points to the simultaneous origin of both meteoritic and terrestrial uranium and potassium. (c) Paneth's own determinations of the ages of iron meteorites, none of which is of a higher order than that now assigned to the earth : here it must be noticed, however, that with the coming into favour of the 'short' time-scale of the universe this item of evidence can no longer be claimed as an argument against an extra-solar origin for iron meteorites.

The chief astronomical arguments bearing on the birth-place of meteorites depend upon observations (a) of the orbits and velocities of falling meteorites; and $(b)$ of the distribution of meteorite falls during the hours of the day. In a few cases heliocentric velocities have been recorded which are sufficiently high to imply an interstellar source for the meteorites concerned. Hoffmeister has, indeed, declared that the interstellar origin of a majority of meteorites is a "well established fact", and the preliminary

* The Origin of Meteorites. By Prof. F. A. Paneth. Pp. $26+3$ plates. (Oxford: Clarendon Press. 1940.) 28. 6d. net. results of Opik's researches at Arizona seem to support this claim. The evidence, however, is conflicting, and the validity of deductions from purely visual observations has been seriously questioned. Paneth refers to recent photographic studies by F. L. Whipple on nine sporadic meteors, which proved that at least eight, and perhaps all, were moving in elliptical orbits; he also cites the opinion of Fletcher Watson and C. C. Wylie that visual observations are inevit. ably subject to a systematic subjective error which always leads to the over-estimating of velocities. Clearly the interstellar hypothesis cannot be regarded as 'well established' and it is still more gravely weakened by the other line of astronomical evidence, which supports the alternative view that meteorites originated in the solar system. It is found that a majority of the meteorites seen to fall strike the earth between noon and midnight, and from this preponderance it can be inferred that most, and possibly all, meteorites move in 'direct' orbits. Niessl has argued against the validity of this inference, but Lindemann has in turn convincingly disposed of Niessl's objection. Summing up the whole position, Paneth thinks we may safely assume that meteorites have been members of the solar system since their formation.

Other points of interest discussed in the lecture refer to the conditions under which meteorites may have been formed and to their subsequent history. Recent attempts to reproduce the Widmanstätten structure show that it is not formed during solidification from a molten mass; but is due to a later re-orientation of the iron and nickel atoms which occurs while the crystals very slowly cool. The perfection of this structure in iron meteorites implies extremely slow cooling at a relatively high temperature The parent mass or masses must therefore have had very considerable dimensions, an inference which is consistent with the common view that meteorites may be fragments of asteroids (or of a single asteroid) which came, perhaps several times, within the Roche limit of, say, Jupiter. C. C. Wylie has found that the Pultusk meteorites moved in elliptical orbits resembling those of the small asteroids in their eccentricities, inclinations to the ecliptic, periods of revolution, and mean distances from the sun. The widely varied 'ages' of iron meteorites further suggest that some of the asteroids or their fragments may have passed near enough to the sun to have had their earlier store of helium driven off.

The long and "rather wild" history of stone meteorites which can be read from their intricate structures also points to periodic heating and cooling, and this again suggests close approach, several times repeated, to the sun. Paneth does not overlook the tantalizing complications that this possibility introduces with regard to the development of the Widmanstätten structure. These and other points of detail - such as the origin of chondrules-continue to remain obscure.

Nevertheless, while admitting that the complete story still remains to be told, it is clear that the leading features of the origin of meteorites are now well within our grasp. Prof. Paneth is to be congratulated on a masterly review of a subject to which he himself has made notable and far-reaching contributions. 